\title{
Orientational dependence of current through molecular films
}

\author{
P.E. Kornilovitch and A.M. Bratkovsky \\ Hewlett-Packard Laboratories, 1501 Page Mill Road, Palo Alto, California 94304
}

(February 16, 2001)

\begin{abstract}
We study the current through molecular films as a function of orientation of the molecules in the film with respect to electrodes. It may change by more than an order of magnitude, depending on the angle between the axis of the molecules and the normal to the electrode. This is a consequence of a strong directional character of $p$-orbitals that determines the conductance through the molecules. We demonstrate this result on an exactly solvable model, and present the calculations for two different experimentally accessible molecular films sandwiched between gold electrodes.
\end{abstract}

PACS numbers: 73.40.Gk, 73.61.Ph, 73.63.Rt

The studies of organic molecules as possible electronic components and devices have grown out of the initial suggestion by Aviram and Ratner [1] into a very active area of research, see e.g. [2, 3]. One of the most important issues in this problem is the role of the moleculesurface interface. The geometry of the contacts [ 6 , the type of binding [5, :87, and the molecule-electrode distance [5.9, 10] were all found to affect the conductance significantly. In this paper we perform a model study of the effect of mutual orientation of the molecules and the electrodes on conductance. We find that the orientational dependence of the current through molecular films is much stronger than reported previously in Ref. [10], and it should be taken into consideration in both interpreting experimental data and designing possible moletronic devices.

The simple argument in favor of a substantial orientational dependence of the conductance is the large anisotropy of the molecule-electrode coupling. In most molecules that have been studied experimentally or theoretically so far, the conduction is due to $\pi$-conjugated molecular orbitals (MOs). Such MOs extend over the whole molecule and facilitate the transport of electrons between the two electrodes. Since those MOs are made mostly of anisotropic $p$ atomic orbitals, the overlap of the conducting MOs with electrode wave functions strongly depends on the angle between the main axis of the molecule and the surface normal. In general, we expect the overlap and the full conductance to be maximal when the lobes of the $p$-orbital of the end atom at the molecule are oriented perpendicular to the surface, and smaller otherwise, as dictated by the symmetry. An estimate of the effect can be made from the general properties of the $p$-orbitals. The overlap integrals of a $p$-orbital with orbitals of other types differ by a factor about 3 to 4 for the two orientations 11. Since the conductance is proportional to the square of the matrix element, which contains a product of two metal-molecule hopping integrals, the total conductance variation with overall geom- etry may therefore reach two orders of magnitude, and in special cases be even larger. This conclusion would be only valid when the electronic structure of the molecule and the molecule-electrode distance does not change significantly while its orientation is being changed. One can anticipate this to apply when the coupling to the electrode is weak, as might be the case in some experimental setups. The calculations 12 suggest that there are attachements of particular molecules with very little resistance to the rotation with respect to metallic surface. The present effect (change of the geometry of the metalmolecule contact) should be distinguished from other geometrical effects caused by intramolecular conformation changes. One possibility, discussed in Ref. [13], is to rotate a segment of a $\pi$-conjugated molecular wire. That would break the conjugation and result in decreased conductance. Another would be to rotate an active part of the molecule with respect to the rest of it to effectively change the current path, which leads to significant changes in resistance of the effective circuit [14].

In order to illustrate the geometric effect on current we shall first consider a simple model shown in Fig. 1 (top panel). A molecular film is sandwiched between two three-dimensional electrodes, which are assumed to have a simple cubic structure with one $s$-orbital per site and the onsite energy $\epsilon_{s}$, which we take as an energy origin. We assume, without loss of generality, that the band in the leads is half-filled, so that the Fermi level $E_{F}=\epsilon_{s}=0$. The hopping integral between the neighbors is $-t_{s}$. The "molecules" have only two $p \pi$-type atomic orbitals with the onsite energies $\epsilon_{p}$ and hopping between those $\pi$-orbitals is $-t_{\pi}$. Those orbitals model $\pi$-conjugated bands of organic conductors. The molecules are allowed to rotate about the bottom "atom" and thereby change the angle $\theta$ between the main axis of the molecules and the surface normal. As $\theta$ changes, the $p$-orbitals remain oriented perpendicular to the molecular "backbone". Because of that, the matrix element between a molecular $p$-orbital and an electrode $s$-orbital 

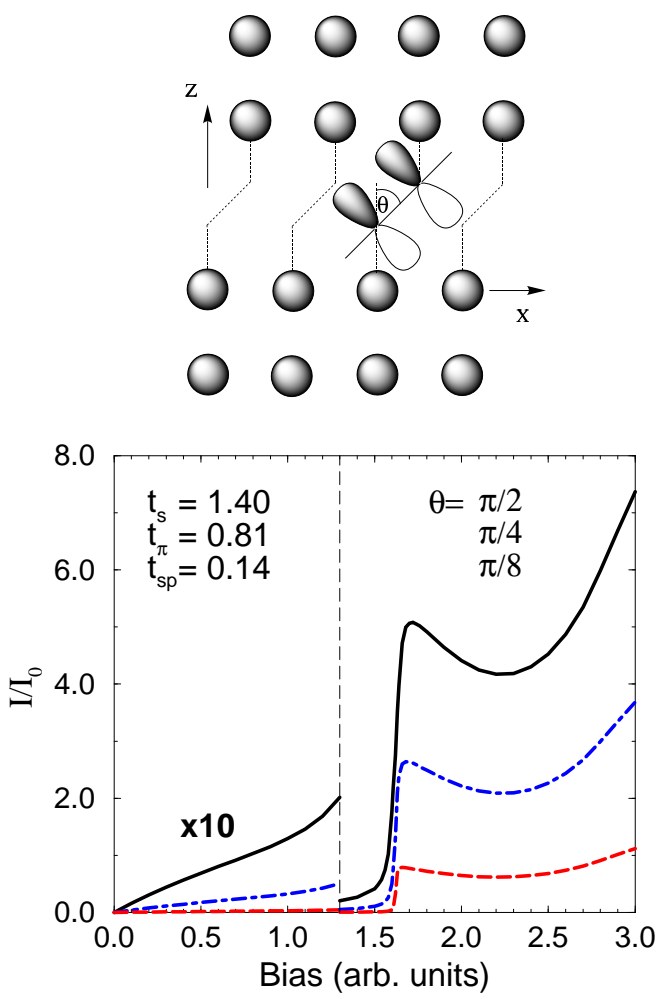

FIG. 1. Top panel: the molecular film between two leads with simple cubic structure and one $s$-orbital per site. Only one molecule of the film is shown explicitly. Due to the symmetry of coupling to the electrodes, the current is zero for the normal orientation $(\theta=0)$ and maximal for the parallel orientation $(\theta=\pi / 2)$ of the molecules. Bottom panel: current per surface unit cell versus bias voltage for the tilt angles $\theta=\pi / 2, \pi / 4$, and $\pi / 8$, with the hopping parameters $t_{s}, t_{\pi}$, and $t_{s p}$ in arbitrary units $\left(t_{0}\right)$. Bias is in units of $t_{0} /|e|$, and the parameter $I_{0}=10^{-3} t_{0} \frac{2 e}{h}$.

varies as $t_{s p} \sin \theta$, where $t_{s p}$ is a constant ( $s p-$ hopping matrix element between the molecule and the electrode). The molecule-electrode bond lengths do not change with $\theta$, and the surfaces of the two electrodes always remain parallel. Each molecule is assumed to have one electron per atom. The distance between the lowest unoccupied MO (LUMO) and highest occupied MO (HOMO), or the "HOMO-LUMO" gap, is $E_{\mathrm{LUMO}}-E_{\mathrm{HOMO}}=2 t_{\pi}$. The middle of the gap is aligned with the chemical potential of the leads at zero bias voltage $V=0$ (meaning that $\left.\epsilon_{p}=\epsilon_{s}=0\right)$.

The current through the film at low temperatures is given by a standard expression [15, 16]

$$
I=\frac{2|e|}{h} \int_{E_{F}-|e| V / 2}^{E_{F}+|e| V / 2} d E \sum_{\vec{k}_{\|}}^{\text {open }}\left|t\left(E, \vec{k}_{\|}\right)\right|^{2},
$$

where the summation goes over the surface Brillouin zone of the lead for open channels, and the transmission co- efficient is found from solution of the scattering problem [16.

$$
t\left(E, \vec{k}_{\|}\right)=i D^{-1} \sqrt{v_{z L} v_{z R}} t_{\pi} t_{s p}^{2} e^{i\left(k_{z L}+k_{z R}\right)} \sin ^{2} \theta
$$

where

$$
\begin{aligned}
D & =\left(E^{2}-t_{\pi}^{2}\right) t_{s}^{2}+E t_{s} t_{s p}^{2}\left(e^{i k_{z L}}+e^{i k_{z R}}\right) \sin ^{2} \theta \\
& +e^{i\left(k_{z L}+k_{z R}\right)} t_{s p}^{4} \sin ^{4} \theta
\end{aligned}
$$

and we assume $\hbar=1$. Then, the band velocities are $v_{z L(R)}=2 t_{s} \sin k_{z L(R)}$, with $k_{z L(R)}$ the z-components of the momenta in the leads, which are found from

$$
2 t_{s} \cos k_{z L(R)}=-E+(-) \frac{|e| V}{2}-2 t_{s}\left(\cos k_{x}+\cos k_{y}\right) .
$$

It is instructive to analyze the transmission in the limiting case of weak coupling to the electrodes, $t_{s p} / t_{\pi} \ll 1$. The exact transmission probability, Eq. (2), acquires the resonant form for zero bias voltage, $V=0$, when $k_{z L}=k_{z R}$ :

$$
\left|t\left(E, \vec{k}_{\|}\right)\right|^{2} \approx \sum_{r=1,2} \frac{\Gamma^{2} / 4}{\left(E-E_{r}\right)^{2}+\Gamma^{2} / 4}
$$

with

$$
\begin{aligned}
E_{1(2)} & =+(-) t_{\pi}-\Delta, \\
\Delta & =\Delta\left(E, \vec{k}_{\|} ; V\right)=\left(t_{s p}^{2} / t_{s}\right) \cos k_{z} \sin ^{2} \theta, \\
\Gamma & =\Gamma\left(E, \vec{k}_{\|} ; V\right)=\left(t_{s p}^{2} / t_{s}\right) \sin k_{z} \sin ^{2} \theta,
\end{aligned}
$$

where $\Delta, \Gamma \ll E_{1}-E_{2}=2 t_{\pi}$ is the shift of the resonant levels, and their width, respectively. For non-zero bias the transmission probability is characterized by different line widths for hopping to the left (right) lead $\Gamma_{L}\left(\Gamma_{R}\right)$, and Eq. (5) will have a general form $\sum_{r=1,2} \frac{\Gamma_{L} \Gamma_{R}}{\left(E-E_{r}\right)^{2}+\Gamma^{2} / 4}$ with $\Gamma=\Gamma_{L}+\Gamma_{R}$. If we were to neglect the dependence of $\Gamma$ on energy and $\vec{k}_{\|}$, we would obtain

$$
I \approx\left\{\begin{array}{cl}
\frac{e^{2}}{h} \frac{\Gamma^{2}}{t_{\pi}^{2}} V \propto \sin ^{4} \theta, & |e| V \ll E_{\mathrm{LUMO}}-E_{\mathrm{HOMO}} \\
\frac{8 \pi|e|}{h} \frac{\Gamma_{L} \Gamma_{R}}{\Gamma_{L}+\Gamma_{R}} \propto \sin ^{2} \theta, & |e| V>E_{\mathrm{LUMO}}-E_{\mathrm{HOMO}} .
\end{array}\right.
$$

The low bias case corresponds to sub-resonant tunneling inside the HOMO-LUMO gap, whereas the case of large bias involves the tunneling through HOMO and LUMO. There is a crossover in the angular dependence of the current through the molecule from $\sin ^{4} \theta$ to $\sin ^{2} \theta$ with the bias. The current saturates at large bias (with further changes due to the density of states in the leads) and its angular dependence becomes less steep. This behavior is confirmed by the results of exact calculations shown in 

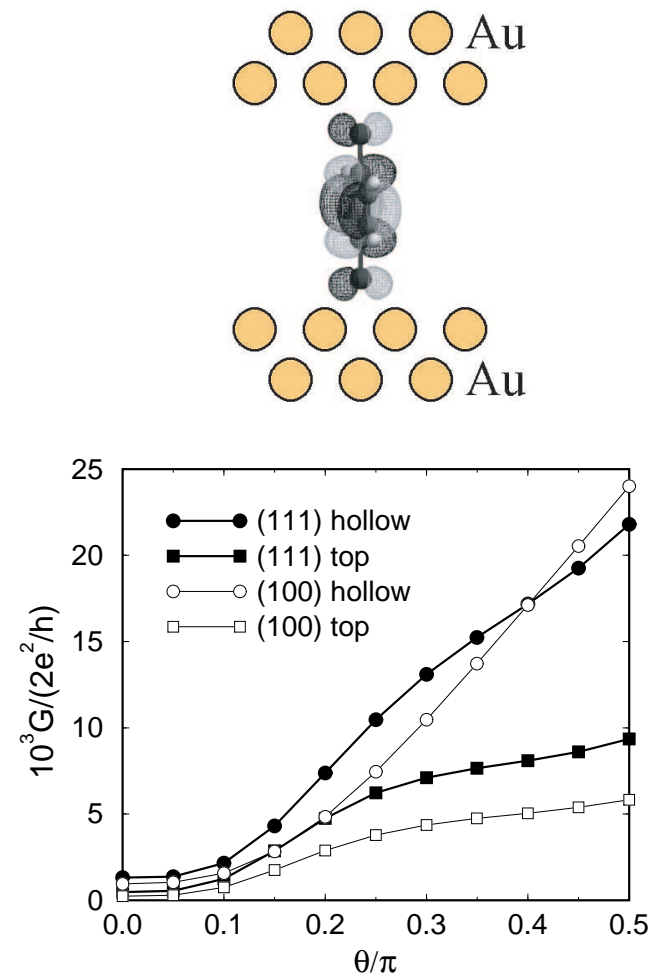

FIG. 2. Top panel: schematic of benzene-(1,4)-dithiolate molecules positioned between two gold electrodes. Also shown is the LUMO of the free molecule found from the density functional theory. Bottom panel: conductance as a function of the tilt angle $\theta$.

Fig. 1. It shows a dependence of current on the angle, which is stronger at small biases. The maximum of the current corresponds to $\theta=\pi / 2$, i.e. to the horizontal orientation of the molecule in the film (backbone of the molecule parallel to the surface of the electrodes). Exact results also show the non-monotonous behavior of the current with the bias, when it is comparable to HOMOLUMO gap, which comes from the complex bias dependence of $k_{z L(R)}$ through the dispersion relations (位).

The formula (5) is, obviously, a particular form of the Breit-Wigner formula and it should apply whenever the width of the resonances is much smaller than the separation between them. Thus, the results, Eq. (9), are more general and should qualitatively apply in more complex molecules too, if the overlap between resonances is not substantial, although it is likely to be violated at high bias voltages.

We illustrate the angular dependence on two types of molecules, benzene-(1,4)-dithiolate $\left(-\mathrm{S}_{-} \mathrm{C}_{6} \mathrm{H}_{4}-\mathrm{S}-\right)$ and $\alpha, \alpha^{\prime}$-xylyl-dithiolate (-S- $\mathrm{CH}_{2}-\mathrm{C}_{6} \mathrm{H}_{4}-\mathrm{CH}_{2}$-S-) placed between two gold electrodes. Both of those systems were studied experimentally 17,18 and theoretically [5, 8, 10, 19,20], but mostly assuming a particular symmet- ric position of the molecules with respect to the surface. Those molecules attach strongly to the gold substrate by thiol, -S-, end groups, which form a chemical bond with $\mathrm{Au}$ [17, 18]. The strong bonding might well mean a substantial change in the electronic structure of the molecule, because of a possible charge flow to/from the molecule bonded to a metal (electron reservoir) due to the difference in their ionization potentials. Therefore, the following calculation is rather the model study to illustrate the orientation effect, which may not be as strong in a particular setup [17,18 compared to our calcualted effect. It is clear, however, that the effect exists and may be large in situations when the coupling to electrodes does not strongly perturb the molecule itself.

In order to compute the conductance, we follow the same general procedure of Ref. [16]. The gold electrodes have been described by a tight binding model with nine $s-, p-$, and $d-$ orbitals per each $\mathrm{Au}$ atom with the parameters from Ref. 21]. The equilibrium molecular geometry is found by the total-energy density functional minimization [22]. The tight-binding parameters for the molecules and molecule-lead interfaces have been taken from the solid-state table of elements [11].

Consider the benzene-(1,4)-dithiolate film. We first place the molecules perpendicular to the gold electrodes, as shown at the top of Fig. 2. Also shown is one of the conducting orbitals, the LUMO. For this orientation the conductance should be small because the $p$-orbitals, that constitute the LUMO (and the HOMO, which also conducts), are parallel to the surface and the overlap is the smallest. Next, we allow the molecules to rotate about one of the end sulfur atoms while keeping the two surfaces parallel and both sulfur-gold bond lengths constant. The molecules are rotated in such a way that the lines through pairs of hydrogen atoms, that are symmetric with respect to molecules' main axis, remain parallel to the gold surfaces. As the angle $\theta$ between the molecules and the surface normal increases, so does the overlap of the sulfur $p$-orbitals with the gold orbitals. Thus, we expect the conductance to grow with $\theta$.

Our numerical results are shown in Fig. 2. We have studied two different surfaces, $\mathrm{Au}(111)$ and $\mathrm{Au}(100)$, and two binding schemes, the "on-top" and the "hollow" positions. In the first case, the sulfur atoms are positioned directly on top of the gold atoms at a distance of $2.39 \AA$ [12]. In the hollow case, the sulfurs are in symmetric positions with respect to three gold atoms of the $\mathrm{Au}(111)$ surface, and with respect to four gold atoms of the $\mathrm{Au}(100)$ surface. In all four cases, we found the conductance to be a steadily increasing function of $\theta$. The ratio $G\left(\frac{\pi}{2}\right) / G(0)$ is the highest $(=26)$ in the $\mathrm{Au}(100)$-hollow case and lowest (=17) in the $\mathrm{Au}(111)$-hollow case. The shapes of all curves are qualitatively similar, while the hollow position is 2 to 3 times more conductive than the top position. Thus we observe a large orientational effect, which magnitude is rather insensitive to the electronic structure 

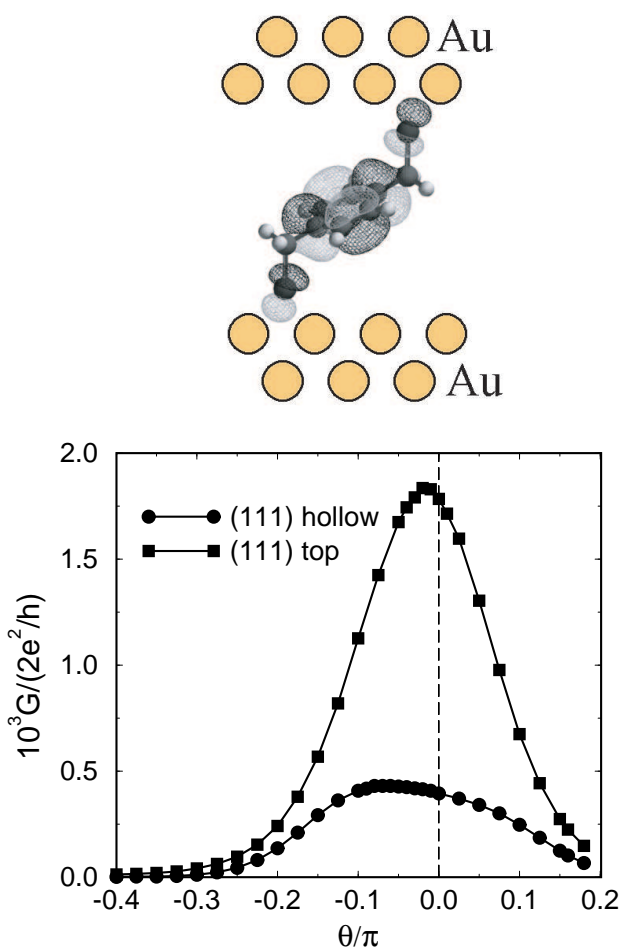

FIG. 3. Top panel: schematic of $\alpha, \alpha^{\prime}$-xylyl-dithiolate molecules placed between two gold electrodes. Also shown is the LUMO of the free molecules computed within the density functional theory. Bottom panel: conductance of the film absorbed on the $\mathrm{Au}(111)$ surfaces.

and geometry of the electrodes. This finding corroborates our main argument that the orientation of molecular $p$ orbitals with respect to the electrodes is an important factor in the problem.

Next, we consider another molecule, $\alpha, \alpha^{\prime}$-xylyldithiolate, see Fig. 3. The $\theta=0$ position is chosen to be the one with the $\mathrm{S}-\mathrm{C}$ bond perpendicular to the gold surface. Then, again, the angle between the $\mathrm{S}-\mathrm{C}$ bond and the surface normal is systematically changed in both directions, while keeping the gold-sulfur bond lengths constant and the two electrode surfaces parallel. Since this molecule is asymmetric with respect to the $\mathrm{S}-\mathrm{C}$ bond, the allowed interval of the tilt angle is also asymmetric, $-0.4 \pi<\theta<0.2 \pi$ (positive angle corresponds to the clockwise rotation of the molecules). We have studied the $\mathrm{Au}(111)$ surface and the two binding positions of sulfur, the on-top and the hollow. The computed conductance is shown at the bottom of Fig. 3. It is maximal at small $\theta$ and falls off upon tilting in either direction. Such a behavior is readily understood if the spatial structure of the conducting MOs is taken into account. In Fig. 3 one of those orbitals, the LUMO, is shown. The hybridization of the sulfur $p$-orbitals with the rest of the molecule has led to the wave function having a preferential direction from the sulfur atoms towards the ring. Still, the wave func- tion retains its $p$-character so that a strong anisotropy of the LUMO-gold overlap is expected. Note that, according to Fig. 3, the precise direction of the wave function is in slight misalignment with the $\mathrm{S}-\mathrm{C}$ bond, which is chosen as $\theta=0$. The maximum overlap, and consequently maximum conductance, is therefore expected for a small negative $\theta$, precisely the behavior we obtained from the full numerical treatment of the scattering problem. This detail indicates the adequacy of the present tight-binding parameterization.

The present results show that the orientational dependence "molecule-electrode" of the conductance is a large effect if the conducting orbitals are extended $\pi$-orbitals, and the contact with electrode does not strongly perturb the molecule itself. The effect derives from the strong directional character of $\pi$-orbitals. We have also studied several other systems of different complexity, both analytically and numerically, and obtained the results consistent with this simple picture. It is usually assumed that the geometry in molecular transport experiments is fixed, but there may be circumstances when the geometry can be changed. Firstly, for some molecule-electrode pairs there may be more than one possible chemisorption mode. For instance, for thiolates on gold it was found in Ref. 12] that the hollow and on-top binding configurations are close in energy but have very different surface-S-C bond angles, $\sim 180^{\circ}$ and $\sim 105^{\circ}$, respectively. Consequently, the molecules may change the configuration with temperature and/or under other external factors. According to our calculations, this may lead to a significant change of conductance. Secondly, the tilt angle in self-assembled monolayers or LangmuirBlodgett films, used in moletronics studies, is never exactly zero [23] and may depend on the temperature, pressure, and/or other parameters of preparation. Thirdly, the angle may change during the measurement process. For instance, in STM probes of single molecules 24 the position of the probing tip is not fixed with respect to the molecule and may record a distribution of conductance if the latter is strongly angle-dependent. Finally, a finite temperature always results in geometry fluctuations that in turn lead to conductance fluctuations. Thus the present mechanism may be one of the sources of the temperature dependence of the conductance.

We thank E. Emberly, A. Onipko, M. Ratner, R.S. Williams, and our colleagues at Quantum Science Research group, Hewlett-Packard Labs, for useful discussions. The work has been partly supported by DARPA.

[1] A. Aviram and M.A. Ratner, Chem. Phys. Lett. 29, 257 (1974). 
[2] Molecular Electronics: Science and Technology, edited by A. Aviram and M.A. Ratner (New York Acad. Sci., New York, 1998).

[3] V. Mujica, A. Nitzan, Y. Mao, W. Davis, M. Kemp, A. Roitberg, and M.A. Ratner, Adv. Chem.Phys. 107, 403 ( 1999).

[4] S.N. Yaliraki and M.A. Ratner, J. Chem. Phys. 109, 5036 (1998).

[5] E.G. Emberly and G. Kirczenow, Phys. Rev. B 58, 10911 (1998).

[6] M. Di Ventra, S.T. Pantelides, and N.D. Lang, Phys. Rev. Lett. 84, 979 (2000).

[7] S.N. Yaliraki, A.E. Roitberg, C. Gonzalez, V. Mujica, and M.A. Ratner, J. Chem. Phys. 111, 6997 (1999).

[8] A. Onipko, Yu. Klimenko, and L. Malysheva, Phys. Rev. B 62, 10480 (2000).

[9] M. Magoga and C. Joachim, Phys. Rev. B 56, 4722 (1997).

[10] S.N. Yaliraki, M. Kemp, and M.A. Ratner, J. Am. Chem. Soc. 121, 3428 (1999).

[11] W.A. Harrison, Electronic structure and the properties of solids (Dover, 1989).

[12] H. Sellers, A. Ulman, Y. Shnidman, and J.E. Eilers, J. Am. Chem. Soc. 115, 9389 (1993).

[13] M. Magoga and C. Joachim, Phys. Rev. B 59, 16011 (1999).

[14] F. Moresco et al., Phys. Rev. Lett. 86, 672 (2001).

[15] R. Landauer, IBM J. Res. Dev. 1, 223 (1957); Phys. Lett. 85A, 91 (1981).

[16] S. Sanvito, C.J. Lambert, J.H. Jefferson, and A.M. Bratkovsky, Phys. Rev. B 59, 11936 (1999).

[17] M.A. Reed, C. Zhou, C.J. Muller, T.P. Burgin, and J.M. Tour, Science 278, 252 (1997).

[18] M. Dorogi, J. Gomez, R. Osifchin, R.P. Andres, and R. Reifenberger, Phys. Rev. B 52, 9071 (1995).

[19] L.E. Hall, J.R. Reimers, N.S. Hush, and K. Silverbrook, J. Chem. Phys. 112, 1510 (2000).

[20] W. Tian, S. Datta, S. Hong, R.G. Reifenberger, J.I. Henderson, and C.P. Kubiak, Physica E 1, 304 (1997); J. Chem. Phys. 109, 2874 (1998).

[21] D.A. Papaconstantopoulos, Handbook of the Band Structure of Elemental Solids (Plenum, New York, 1986).

[22] Spartan version 5.0, Wavefunction, Inc. 18401 Von Karman Avenue, Suite 370, Irvine, CA 92612 U.S.A.

[23] J.M. Tour, L. Jones, D.L. Pearson, J.J.S. Lamba, T.P. Burgin, G.M. Whitesides, D.L. Allara, A.N. Parikh, and S.V. Atre, J. Am. Chem. Soc. 117, 9529 (1995).

[24] L.A. Bumm, J.J. Arnold, M.T. Cygan, T.D. Dunbar, T.P. Burgin, L. Jones II, D.L. Allara, J.M. Tour, and P.S. Weiss, Science 271, 1705 (1996). 